\title{
SISTEMAS DE INNOVACIÓN (LEARNING BY INTERACTING): ANTECEDENTES TEÓRICOS EN LOS APORTES DE GEORGE HERBERT MEAD
}

\section{Innovation Systems (Learning by Interacting): Theoretical Precedents in the Contribution of George Herbert Mead}

Sistemas de inovação (learning by interacting): antecedentes teóricos nos aportes de George Herbert Meade

\section{RESUMEN}

Los procesos de learning by interacting son métodos característicos de los sistemas de innovación, elaborados en el marco del evolucionismo económico. En los sistemas de innovación, el recurso primordial es el conocimiento. Una parte de dicho conocimiento es de orden tácito, y para su aprendizaje se requiere la interacción entre los participantes. En este trabajo, se presentará el pensamiento sobre el interaccionismo simbólico del pragmatista G. H. Mead. Sus ideas brindan elementos de interés para incorporar a los procesos de learnign by interacting, $y$ también a otras áreas de la creatividad y de la innovación.

\begin{abstract}
Learning by interacting processes are methods that characterize innovation systems carried out within the frame of economic evolution. Knowledge is the main resource within innovation systems. Part of this knowledge is unspoken and in order to learn it, it is necessary for participants to interact. This paper presents the idea that the pragmatist G. H. Mead had regarding symbolic interactionism. His ideas provide us with elements of interest to incorporate within learning by interacting processes and other creativity and innovation areas.
\end{abstract}

KEYWORDS: E-learning, interaction, creativity, innovation.

\section{RESUMO}

Os processos de learning by interacting são métodos característicos dos sistemas de inovação, elaborados no marco do evolucionismo económico. Nos sistemas de inovação, o recurso primordial é o conhecimento. Uma parte de dito conhecimento é de ordem tácita, e para o seu aprendizado se requer a interação entre os participantes. Em este trabalho, se apresentará o pensamento sobre o interacionismo simbólico do pragmatista G. H. Meade. As suas ideias oferecem elementos de interesse para incorporar aos processos de learnign by interacting, e também a outras áreas da criatividade e da inovação.

PalaVRas CHAVE: e-learning, interação, criatividade, inovação. 
INTRODUCCIÓN

Dentro del evolucionismo económico, se conforma el modelo de los sistemas de innovación que pueden ser definidos en dos sentidos:

The narrow definition would include organizations and institutions involved in searching and exploring — such as R\&D departments, technological institutes and universities-. The broad definition which follows from the theoretical perspective presented above include all parts and aspects of the economic structure and the institutional set up affecting learning as well as searching and exploring (Lundvall, 1992, p. 13).

En estos sistemas, el recurso primordial es el conocimiento, y el aprendizaje es el proceso más importante para generar innovación. Una de sus características es el proceso de learning by interacting (aprendizaje por interacción), como uno de los mecanismos generadores de innovaciones. Luego de las propuestas de Arrow (1962) sobre learning by doing (aprender haciendo) y de Rosenberg (1982) acerca del learning by using (aprender con el uso), autores como Lundvall incorporan el concepto de learning by interacting (aprendizaje por interacción) como una característica para determinados tipos de procesos de innovación.

En diversos trabajos, elaborados en el ámbito de los sistemas nacionales de innovación, autores como Johnson, Edquist y Lundvall (2003), Lundvall (2005a) y Lundvall (2007) mencionan las obras de George Herbert Mead, de la filosofía pragmatista y de la teoría sociológica del interaccionismo simbólico como fuentes teóricas de los procesos de learning by interacting.

El presente trabajo tiene por objetivo analizar los aportes teóricos de George Herbert Mead, sus predecesores y del interaccionismo simbólico, para comprender más adecuadamente aquellos procesos de innovación basados en el aprendizaje por interacción.

En primer lugar, se presentarán las influencias recibidas por Mead, en particular del pragmatismo estadounidense —movimiento del cual formó parte—,y de otros autores, como Charles Cooley y Wilhem Wundt. En segundo término, se detallarán los principales aspectos de la teoría elaborada por Mead, que servirá de base al interaccionismo simbólico. Finalmente, se efectuarán la conclusiones, que explican la utilidad del pensamiento de Mead dentro de los sistemas de innovación del evolucionismo económico, así como en los procesos de creatividad por interacción.

\section{EL PRAGMATISMO AMERICANO}

George Herbert Mead (1863-1931), filósofo relacionado con el pragmatismo estadounidense, y psicólogo social, desarrolló los aportes teóricos base de la corriente sociológica del interaccionismo simbólico, iniciada por su discípulo Herbert Blumer. Mead es considerado el cuarto miembro más relevante de la tradición de la filosofía pragmática estadounidense, junto con Charles Sanders Peirce, William James y John Dewey. Peirce y James fueron los iniciadores de esta tradición, mientras que Dewey y Mead en una segunda etapa realizaron modificaciones a las ideas precursoras (Rescher, 2012).

Los autores iniciales de la filosofía pragmatista -Peirce y James - con diferentes tipos de influencia cada uno de ellos tuvieron en común los aportes de Chauncey Wright, cuyas principales ideas se reseñan a continuación.

Chauncey Wright (1830-1875), matemático y filósofo estadounidense, que apoyó la tesis de la teoría evolucionista, colaboró con Charles Darwin en sus trabajos de investigación (Maden y Maden, 1952). En 1872, al reunirse ambos investigadores, Darwin le solicitó colaboración en el siguiente problema: conseguir explicar en términos evolutivos el desarrollo físico del hombre y los animales y el despliegue del instinto animal hasta alcanzar los grados más altos de la inteligencia y de la razón humana (Sini, 1999).

Al respecto, en 1873, Wright elaboró un ensayo, "The Evolution of self-Consciousness", que expone su teoría del origen y el desarrollo del lenguaje. Se inicia con el uso de gestos instintivos, sin vocación comunicativa, y evoluciona hacia los gestos verbales resultantes de una acción social entre los participantes de los grupos cuya intención es comunicarse.

Centrado en el lenguaje, Wright estudia el desarrollo de la autoconciencia humana: los animales con una conciencia animal hacen un uso inconsciente de los signos. Por otra parte, el hombre los utiliza en forma
Sistemas de

innovación

(learning by

interacting):

antecedentes

teóricos en

los aportes de

George Herbert

Mead 
consciente, al reconocerlos y ser capaces de reflexionar con ellos y sobre ellos. Esta capacidad de reflexión es el resultado del pensamiento autoconsciente, que refleja la inteligencia humana (Parravicini, 2009). Por esta vía, Osvaldo Wright avanzaba en la resolución del "problema de Retondaro I Darwin". Las ideas del evolucionismo de Darwin influyeron en los distintos filósofos pragmatistas en un sentido amplio. Los desarrollos de Wright sobre el lenguaje se reflejarán en la semiótica de C. S. Peirce y con mayor claridad en los trabajos de G. H. Mead, que se presentarán a posteriori.

Charles Sander Peirce (1839-1914) fue el iniciador de la corriente filosófica pragmatista (Peirce, 1878). Era un científico en el campo de la astronomía, la óptica, la química y las matemáticas; se acercó, en primer lugar, a la epistemología y la lógica, derivando en la lógica simbólica (fue junto con Ferdinand de Saussure uno de los precursores de la semiótica).

Sus comienzos como estudioso de las ciencias naturales, campo al que contribuyó con diversas investigaciones, explican su interés por trasladarlas al campo de la epistemología y la lógica. Consideraba que la filosofía debía actualizarse metodológicamente, con métodos científicos como procesos para acercarse a la verdad.

El pragmatismo de Peirce, en primer término, se puede definir como un sistema por el cual el significado de una concepción intelectual está determinado por las consecuencias prácticas que tiene dicho concepto. La máxima del pragmatismo es:

It appears, then, that the rule for attaining the third grade of clearness of apprehension is as follows: Consider what effects, that might conceivably have practical bearings, we conceive the object of our conception to have. Then, our conception of these effects is the whole of our conception of the object (Peirce, 1878, p. 293).

El método de la ciencia está basado en los experimentos, en busca de la verdad, construida juntamente con la comunidad de científicos. Este proceso da lugar a nuevo conocimiento, como parte de la creatividad en la ciencia. A tal fin Peirce proponía estudiar los actos - experiencias-, superando los dualismos entre sujeto y objeto, considerando la aceptación de creencias — una forma de verdades intermedias - como instrumento generador 64 | de hábitos temporales para desenvolverse en el mundo.
En cuanto a los métodos de investigación en filosofía, se centraba en los análisis de la lógica, con aportaciones que ampliaban dicho campo. $\mathrm{Al}$ respecto, se reseñan tres cuestiones:

1. Propuesta del método de la abducción como complementario a la inducción y a la deducción, para desarrollar una lógica de la investigación. La abducción era el método creativo más efectivo para generar nuevo conocimiento y nuevas hipótesis, que permitían remover creencias temporales dentro del proceso de conocimiento hacia verdades ulteriores. En palabras del autor: "Cada punto de la teoría científica que hoy aparece como establecido, se debe a la abducción" (Peirce, 1903, p. 172). Peirce consideraba que la realidad no estaba determinada sino en evolución, pasando de una visión estática a una dinámica. Frente a un estricto determinismo, sostuvo que el mundo tiene un carácter indefinido. Estas indefiniciones de la realidad permitían un amplio campo para la creatividad y para la investigación de nuevas verdades mediante el método de la abducción. (Nubiola, 2001; Barrena, 2008).

2. Definición de la lógica como la ciencia de los signos que centra sus estudios en la semiosis que tendrá influencia en los trabajos de Mead. Peirce definió tres ramas de la semiótica: la gramática especulativa, la lógica pura y la metodéutica o retórica, donde un signo da nacimiento a otro signo. La experiencia que permite construir el pensamiento está mediatizada por interpretaciones relacionadas con inferencias de signos que las representan. Por tanto, todo pensamiento está mediado, es una interpretación de los hechos, por medio de los signos. Nunca se percibe o se piensa en el mundo en forma directa, sino a través de los signos, pues todo pensamiento es signo.

3. Finalmente considera que la búsqueda de la verdad es un proceso que necesita en primer término la falibilidad. Dado que todo conocimiento puede ser en principio erróneo, cabe la posibilidad de negar una proposición, obteniendo una nueva certeza sobre lo conocido, al cambiar su valor de verdad. En segundo término, de la acción cooperativa entre los miembros de la comunidad de investigadores; los problemas por resolver son propios de la comunidad científica. En estos procesos de investigación, la comunidad científica evidencia consenso alcanzado de forma no coactiva, propuestas que deberían ser replicadas en los ámbitos de la filosofía (Bernstein, 1993).

William James (1842-1910) se destacó por la difusión que hizo del pragmatismo, reconociendo a Peirce como iniciador de este movimiento. Comenzó su trayectoria académica como profesor de Fisiología en la Universidad de Harvard, para luego ocupar la cátedra de Psicología y dirigir el primer centro de psicología experimental — de acuerdo con lo aprendido con W. Wundt en Leipzig—, destacándose su tratado de 1890 "Principles of Psychology". Define la experiencia en relación con el proceso de conducta, siguiendo los principios de la 
escuela psicológica funcionalista y del conductismo que se centran en el acto observable.

Una de las influencias del pensamiento psicológico de James en Mead se encuentra en el paralelismo de la idea de la personalidad de James conformada por tres partes: el mí material (cuerpo), el mí social (las personas del entorno) y el mí espiritual (ideas, pensamientos), y la propuesta de conductismo social de Mead (Rizo, 2008).

Con posterioridad a sus intereses en psicología — sobre fines del siglo XIX-, James desarrolló los aportes en filosofía dentro del pragmatismo, reflejando la influencia recibida del evolucionismo y su posición pluralista de la verdad (James, 1898). La teoría de la verdad se relaciona con su postura de empirismo radical, desplazando el centro del pragmatismo desde una ciencia del conocimiento, como significaba para Peirce, hacia una aplicación del conocimiento, útil para la acción. Para James, el pragmatismo es un método, donde la obtención de verdades tiene por finalidad una adecuación a la realidad. La realidad es un proceso dinámico continuo, de experiencias en constante cambio. El objetivo del pensamiento humano es obtener creencias que se convertirán en reglas para la acción, en hábitos que sirvan para guiar la conducta en un medio con variaciones azarosas e indeterminadas. Las verdades son históricas tanto en las ciencias naturales como en la filosofía, se hallan siempre bajo revisión, evolucionan en relación con los cambios del contexto y se desarrollan en forma continua. La verdad es una adecuación a la realidad de manera práctica y dinámica, es un proceso abierto, donde se verifican, validan y modifican, en cuanto a su aplicación en la acción.

Las creencias obtenidas, al ser guías para la conducta, deben ser socialmente consensuadas, pues la acción es en gran medida interacción con otros que deben compartir el significado de dichos actos, sin la cual no habría comunicación. Por tanto, James amplía el concepto de Peirce de creencias compartidas por la comunidad de investigadores, que deben ser ahora verdades relativas a toda la sociedad. Una persona no puede imponer una verdad a los demás miembros de la sociedad, quienes sí podrían aceptarla luego de haberla verificado en sus propias experiencias.

La segunda etapa pragmatista, integrada por Dewey y Mead, supuso nuevos planteamientos en esta corriente de pensamiento, que se centró en una teoría de la acción, junto con una actividad social y política de los filósofos, como se explicitará más adelante.

John Dewey (1859-1952) fue el filósofo pragmatista que tuvo más influencia en Mead, debido en gran parte a la relación personal que mantuvieron como docentes en la Michigan y en la Chicago University. Este filósofo, que en sus inicios se enmarcaba dentro del pensamiento del idealismo hegeliano, incorporó las teorías de Darwin y asumió una filosofía de características evolucionistas, con base biológica y reconociendo la experimentación científica. Finalmente, denominó su propuesta instrumentalismo naturalista. Instrumentalismo, pues consideró que pensar es un instrumento para la acción, por ende, los pensamientos tienen valor instrumental; y naturalista en relación con la ideas biologistas de la evolución darwiniana.

Dewey se adhirió al pragmatismo, manteniendo determinados principios elaborados por Peirce y James, y abordando luego nuevos enfoques y temáticas. Compartió, entre otras cuestiones, la importancia del método científico para actualizar la filosofía, destacando el aspecto central de los experimentos en dichos procesos en busca de la verdad. A partir de un enfoque evolucionista, reconoce una realidad cambiante que conlleva una búsqueda constante de la verdad, derivando en creencias de orden temporal. Se basaba en la idea de falibilismo $y$ en la necesidad de consensuar en forma libre las verdades socialmente, para que ellas sean hábitos de acción e interacción social. Estas verdades que serán consensuadas socialmente requerían que los participantes de la sociedad tuviesen los elementos y las condiciones necesarias para progresar en las investigaciones, e incorporar los hábitos en busca de un crecimiento constante. Para el ideal de esa sociedad, el desarrollo educativo y la democracia plena eran cuestiones fundamentales.

Para Dewey, la implementación de hábitos inteligentes en la educación de los niños era esencial, por lo que sus trabajos fueron reconocidos y llevados a la práctica en su época. Proponía los mismos principios que para el desarrollo de la filosofía, basarse en la libre y activa experimentación de los alumnos —en el contexto de la interacción social y con el entorno físico- como medio para la búsqueda de la verdad (Dewey, 1903).
Sistemas de innovación (learning by interacting): antecedentes teóricos en los aportes de George Herbert Mead
I Panorama

| pp.62-72

I Volumen 9

I Número 17 | Julio-diciembre | 2015 
Por otra parte, el sistema democrático para Dewey era un medio y un fin para el crecimiento de la sociedad. Como la realidad es esencialmente dinámica, se debe contar con un sistema político abierto y flexible que incorpore la imprevisibilidad y el cambio en forma consensuada, y este no era otro que una democracia liberal, con plena participación de todos los sectores. Por ello, entre otras iniciativas, proponía el derecho al voto femenino, y fue uno de los primeros miembros de la Asociación Nacional para el Avance de la Gente de Color.

Dewey explica las ventajas del método científico y analiza los cambios acaecidos en el ámbito tecnológico a partir de la revolución industrial y, en lo social, por la instrumentación de nuevos métodos en educación y nuevas formas de relaciones humanas (Dewey, 1930).

Asimismo, continuó la tradición de explicar características de la naturaleza humana a la luz de la teoría evolucionista. Estudió desde la psicología la importancia de la conciencia en la acción social, como proceso interno al hombre, que media entre un estímulo y la conducta final. De esta forma, la conciencia asume un papel causal en el comportamiento del hombre y permite estudiar los efectos de la autoconciencia en los procesos de interacción social, elemento de utilidad en los análisis posteriores de Mead.

\section{Charles Cooley y WILHeIM WUNDT}

Charles Horton Cooley (1864-1929) fue un economista y sociólogo estadounidense, que compartió con Mead el ámbito docente de la Universidad de Michigan entre 1891 y 1894, cuyas ideas influyeron en la obra posterior del interaccionismo simbólico de Mead.

Uno de los aportes de Cooley que mayor impacto tuvo en la obra de Mead fue el concepto del self looking glass

Panorama I

pp. 62-72।

Volumen 9 I

Número 17 | ser humano que se define como la posibilidad de tratar de entender al otro y comprender lo que le está sucediendo. En segundo lugar, detalla la presencia del yo espejo que consiste en incorporar la mirada del otro en nosotros mismos; es como la presencia de la conciencia social en nuestra identidad. De esta forma, el individuo tiene dos componentes internos que lo habitan, como un "yo desdoblado", que entran en diálogo de forma previa a la acción (Cooley, 1902).

Cooley con la idea del yo espejo observa que la imagen que transmiten los otros sobre uno mismo finalmente forma parte de nuestra identidad, por tanto, el ámbito social determinaría al individuo.

Ideas similares ya habían sido desarrolladas por Adam Smith en su obra La teoría de los sentimientos morales" (Smith, 1997), donde la visión del espectador imparcial generada por Smith (Jacobs, 2006) se asemejará a la idea del self-looking glass de Cooley. Según Smith, las personas mantienen relaciones sociales de mutuo acuerdo sin necesidad de árbitros externos, por la existencia del árbitro interno. Dicho árbitro interno se conforma de tres elementos: en primer lugar, su capacidad racional; en segundo término, la simpatía; y a partir de la capacidad de simpatía, se construye el tercer elemento, que es el espectador imparcial, que pasa por incorporar al Otro en nosotros, es una conciencia que introyecta en nuestro yo, la mirada del Otro.

Wilheim Wundt (1832-1920) es un psicólogo alemán, reconocido como el fundador de la psicología experimental. En su laboratorio de la Universidad de Leipzig, Wundt utilizó los métodos de las ciencias físicas para el estudio de los procesos conscientes o experiencias inmediatas. En este ámbito universitario, continuó su formación G. H. Mead, luego de estudiar en Harvard (Joas, 1978).

El aporte de Wundt al estudio de los gestos, entre otros temas, tuvo influencia sobre la obra de Mead, quien estudia los gestos no verbales como el primer eslabón en el proceso de interacción social humana, idea en parte ya anticipada por C. Wright.

Mead en su obra Espiritu, persona y sociedad inicia el capítulo 7, "Wundt y el concepto de gesto", explicando que "Wundt aisló una valiosísima concepción del gesto que más tarde lo convierte en un símbolo, pero que se descubre en sus primeras etapas como una parte de un acto social (Mead, 1982, p. 85). 
Los gestos no solo son considerados como la expresión de las emociones, en el sentido dado por Darwin, sino que Wundt los desarrolla también como instancias primarias de interacciones sociales. Ante un gesto no verbal el/los otro/s participante/s de la acción generan algún tipo de reacción. Mead, siguiendo los planteamientos de Wundt, considera que frente a los gestos no verbales se debe actuar inmediatamente: "Tiene que adaptarse 'instintivamente' a la actitud del otro individuo” (1982, p. 86).

Luego Mead trata los gestos vocales, característicos del ser humano: "Ahora bien, cuando ese gesto representa la idea que hay detrás de él y provoca esa idea en el otro individuo, entonces tenemos un símbolo significante" (p. 87). Continúa la evolución desde los gestos no verbales a los gestos verbales, para explicar el paso desde los comportamientos instintivos a una reflexividad en la acción comunicativa que evidenciará la existencia de la inteligencia.

\section{EL PENSAMIENTO DE GEORGE HERBERT MEAD}

George Herbert Mead fue un filósofo del movimiento pragmatista estadounidense, influenciado por las teorías evolucionistas de Ch. Darwin, y además un psicólogo social, quien con Gabriel Tarde fueron los precursores de la psicología social. Mead, junto con Dewey, tal como se anticipó, fueron los filósofos de la segunda etapa del pragmatismo, etapa que se centra en la teoría de la acción, como explica Sánchez de la Yncera (1990, p. 54):

$$
\begin{aligned}
& \text { E1 planteamiento general incluye las } \\
& \text { posiciones de Peirce, James, Dewey y } \\
& \text { Mead. Se basa en la aceptación del método } \\
& \text { científico en un contexto no problemático y } \\
& \text { ofrece una teoría del significado y una teoría } \\
& \text { de la verdad. El otro es un planteamiento } \\
& \text { más específico, desarrollado por Dewey y por } \\
& \text { Mead y que ofrece una filosofía de la vida, } \\
& \text { una filosofía de la reconstrucción social que } \\
& \text { acentúa la importancia de la acción humana } \\
& \text { para hacer del mundo un lugar mejor. }
\end{aligned}
$$

Asimismo tanto Mead como Dewey asumen un compromiso de acción social y político, que deriva de su ideal de sociedad:

La idea política que se halla en el trasfondo del pragmatismo en general es la del liberalismo y su intención política es la de fundamentar la armonía del individuo con la sociedad... El liberalismo democrático encuentra su expresión en el intento de Mead de anclar la racionalidad del individuo en la racionalidad de la sociedad haciendo así posible la armonía entre ambos (Carabaña y Lamo de Espinosa, 1978, p. 159).

El pensamiento de Mead puede abordarse desde diferentes puntos de vista, en particular es interesante verlo desde el punto de vista de la creatividad. Al respecto asegura Hans Joas:

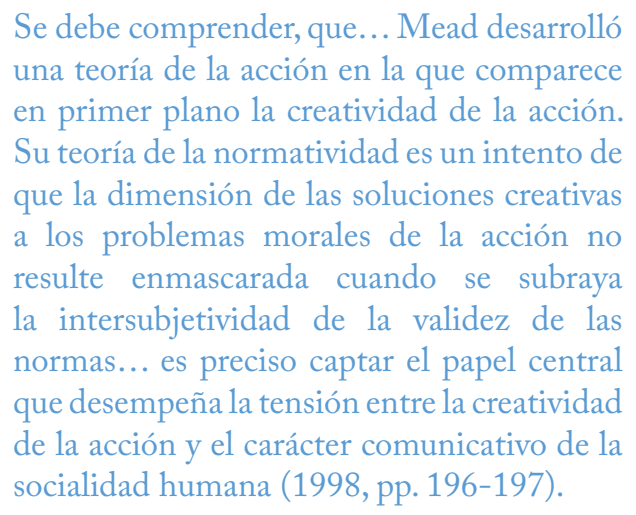

A continuación, se presenta un análisis de las ideas de Mead para una comprensión del interaccionismo simbólico y del learning by interacting de los economistas evolucionistas. Mead explica la relación equilibrada entre individuo y sociedad, a través de una teoría de la acción y de una teoría del control social reflexivo, que deriva en una dinámica positiva entre creatividad y autocontrol. Une la teoría de la acción con la del control social, lo cual significa que la creatividad, la innovación y la diferenciación se conjugan con la reiteración y el orden social. En este sentido, se identificarán individuos que actúan en forma creativa, por una parte, y que tienen un control social de orden autorregulado, por otra parte.

A continuación, se presentará el estudio de la interrelación entre el individuo y la sociedad — teoría de la acción y propuesta del autocontrol social- como un tema central de la obra de Mead, y se explicará dicho proceso mediante dos aspectos:

1. El lenguaje como elemento central.

2. La interrelación social y las etapas de la constitución del self.

I Panorama

I pp. 62-72

I Volumen 9

I Número 17

I Julio-diciembre

| 2015 
EL LENGUAJE COMO ELEMENTO CENTRAL

Los principios básicos que explican esta relación individuo-sociedad se hallan en el uso del lenguaje y en la interpretación de símbolos, siguiendo la influencia de los estudios pioneros de la semiosis de Charles Sanders Peirce.

Como se explicó previamente, Mead, siguiendo el pensamiento de Wundt, inició el estudio de la evolución de la comunicación con los gestos no verbales, de orden instintivo, que se pueden hallar tanto en animales como en seres humanos. Luego avanzó en los gestos verbales, que contemplan la presencia de los símbolos significantes.

La existencia del espíritu y de la inteligencia solo es posible según gestos como símbolos-significantes, porque solo según gestos que son símbolos significantes puede existir el pensamiento, que es simplemente una conversación subjetivada o implícita del individuo consigo mismo por medio de tales gestos. La internalización en nuestra experiencia de las conversaciones de gestos externos que llevamos a cabo con otros individuos en el proceso social es la esencia del pensamiento (Mead, 1982, p. 90).

El gesto no verbal, de orden instintivo, evoluciona hacia el gesto verbal propiamente humano, que incluye un tipo de comunicación de un símbolo y que va a tener significado cuando el individuo sea capaz de tomar el papel del otro. Dentro del proceso enunciado, surge la mente que internaliza la conversación externa de gestos significantes. Así, el desempeño de papeles supone, en primera instancia, ser capaz de tomar el papel del otro y concebirse a sí mismo como un objeto de análisis.

La utilización de gestos verbales que contemplan símbolos significantes tiene al menos cuatro aspectos por considerar:

Volumen 91

Número 17 |

2. El proceso reflexivo de diálogo interno se explica por la presencia del otro en mí. Esta construcción responde a las influencias de C. Cooley y de A. Smith, con la idea del yo espejo y el espectador imparcial, respectivamente, derivados a su vez del concepto de simpatía. De esta forma, mi acción social sería de orden autorregulada, ante la presencia de la conciencia social en mi individualidad.

3. El individuo introyecta de la sociedad los símbolos utilizados, pero también dispone de la posibilidad de resignificarlos. Mead explica que se hace un uso diferente del lenguaje común existente en cada contacto nuevo entre personas; ese elemento de novedad se debe a la reacción que los individuos plantean hacia el grupo al cual pertenecen (Mead, 1982). Los seres humanos tenemos un componente creativo, que no queda anulado por la sociedad circundante. En un diálogo interno previo a emitir el mensaje, se tiene la potencialidad de introducir variaciones en los significados y disponerlos en forma innovadora. Estos aportes individuales en nuevas significaciones explican el proceso evolutivo de los lenguajes a lo largo del tiempo.

Mead brinda su resolución al problema de Darwin demostrando cómo, a partir del instinto, los seres humanos evolucionan hacia un comportamiento racional, mediante el uso del lenguaje (siguiendo las ideas de Wright). Darles significado a los signos que se utilizarán, con un arreglo de medios a fines, implica reflexión $y$, por ende, pensamiento, lo que refleja la presencia de la inteligencia humana. Significar a un signo implica pensar: "El pensamiento es simplemente el razonamiento del individuo, una conversación entre lo que he llamado el “yo" y el “mí” (Mead, 1982, p. 343).

\section{LA INTERRELACIÓN SOCLAL Y LAS ETAPAS DE LA CONSTI-} TUCIÓN DEL SELF

El self constará de dos elementos centrales: el mí y el yo, aspectos en gran medida desarrollados en la obra de psicología de William James. El self es, fundamentalmente, la capacidad de considerarse a uno mismo como objeto, pero, a la vez, formar parte del sujeto. Si bien es un proceso íntimamente relacionado con el desarrollo de la mente, es consecuencia de un proceso social; se basa en la comunicación y la interacción entre los humanos. El mecanismo general para el desarrollo del self es la reflexión o la capacidad de ponerse en el lugar de un otro individualizado - una personalidad que se considere significativa-, el Otro significante, o de un otro generalizado - el conjunto de normas y valores del colectivo en que el individuo desarrolla su existencia-, $\mathrm{y}$ actuar en parte como se espera que lo haga y en parte respondiendo a la propia iniciativa.
Para Mead, los otros significativos son las personas con las cuales el individuo comparte más estrechamente sus 
relaciones y cuyas opiniones valora de manera especial. A medida que transcurre su vida, el individuo amplía sus otros significativos, desarrollando distintos tipos de relación con cada uno de ellos, lo cual genera la creación de varios "sí mismos" elementales, creados en virtud de esas relaciones. Sin embargo, cuando el individuo mediante una abstracción piensa en los otros significativos según el conjunto, asoma el “otro generalizado”. De acuerdo con Mead, solo en relación con el otro generalizado es que surge el "sí mismo completo". En cuanto una persona desarrolle este self, se convierte en miembro efectivo de una sociedad.

Mead explica que el niño en el momento de nace carece de self, y que este se adquiere gracias a la interacción social, la relación y la comunicación con los otros. El niño puede conferir sentido a sus comportamientos a partir de las interpretaciones que recibe de los otros, cuando estos reaccionan a sus actos.

Mead diferencia dos fases dentro del proceso evolutivo de desarrollo del self:

1. Etapa del juego (play): es aquella en la que el niño aprende a adoptar la perspectiva de "otro". A través del juego, y concretamente en los que se juega a ser "otros", el niño aprende a recibir respuestas y actuar como se espera que lo haga el personaje que está escenificando (un indio, un médico, una madre, una enfermera, etc.).

2. Etapa de juego organizado (game), que consiste en que el sujeto debe empezar a ser consciente de que existe un equipo que espera de él una determinada respuesta congruente con las normas de juego. Ahora los otros jugadores ocupan posiciones funcionales y realizan sus papeles respectivos en forma competitiva, como en un sistema de división del trabajo. Así se gesta la formación del Otro generalizado. El otro generalizado se define como la actitud del conjunto de la comunidad en la que el individuo desarrolla su existencia.

Cuando el individuo logra esta doble capacidad, desarrolla plenamente su self. Mead identifica dos aspectos o fases del self, que llama el yo y el mí. El yo es la respuesta inmediata de un individuo a otro. Es el aspecto más afectivo, irracional, imprevisible y creativo del self; mientras que el mí se define como conjunto organizado de actitudes de los demás que uno asume. El yo sería la parte más personal de cada sujeto, mientras que el mí se corresponde con los valores y las normas adquiridos por la socialización. La manera en que se articulan ambas fases determina la personalidad diferenciada de cada individuo.
Mead entiende al mí como el conjunto de actitudes de los otros que cada persona interioriza como propias. Es decir, para tener conciencia de sí — característica definitoria de la persona-, uno tiene que tener la actitud del otro en su propio organismo. La persona que es capaz de mantenerse en la comunidad es reconocida en esta en la medida en que reconoce a los otros. El mí representa una parte convencional, habitual.

Por su parte, el yo es el componente creativo y que brinda originalidad al self; sin la existencia del yo, los individuos aparecerían completamente sujetos al control social externo e interno -implícito en el mí-; la innovación se localiza en la acción.

El self de cada individuo contiene la creatividad en el componente agencial del yo, que se explica en una teoría de la acción, y el mí que refleja la estructura del otro generalizado, que corresponde al control y la conciencia social.

\section{LOS PRINCIPIOS DEL INTERACCIONISMO SIMBÓLICO}

Si bien se suele correlacionar el término interaccionismo simbólico con G. H. Mead, dicha teoría sociológica fue desarrollada por su discípulo Herbert Blumer. Herbert Blumer (1900-1987), sociólogo estadounidense, perteneciente a la Escuela de Chicago, desarrolla la teoría microsociológica del interaccionimo simbólico, siguiendo la ideas de Mead. En su libro El interaccionismo simbólico (Blumer, 1982) propone los tres principios básicos de dicha teoría, que se hallan en paralelo a las ideas de Mead:

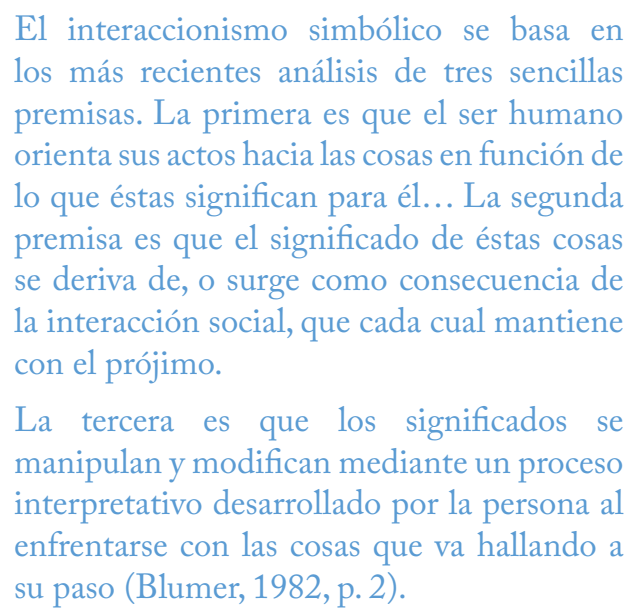

El autor explica dichas premisas en relación con la segunda de ella, que representa la presencia del
Sistemas de

innovación

(learning by

interacting):

antecedentes

teóricos en

los aportes de

George Herbert

Mead 
componente social, el mí en el self del pensamiento de Mead:

Los actos de los demás producen el efecto Osvaldo de definirle la cosa a esa persona. En suma, Retondaro L el interaccionismo simbólico considera que el significado es un producto social, una creación que emana de y a través de las actividades definitorias de los individuos a medida que estos interactúan (Blumer, 1982, p. 3).

Finalmente, la tercera premisa básica es el componente creativo y agencial del yo en el self, que resignifica los símbolos socialmente aprendidos y evidencia el componente autónomo y original del individuo. Al respecto, Blumer comenta:

Mientras que el significado de las cosas se forma en el contexto de la interacción social y es deducido por la persona a través de ésta, sería un error pensar que la utilización del significado por una persona no es sino una aplicación del significado así obtenido... El agente selecciona, verifica, elimina, reagrupa y transforma los significados a tenor de la situación en la que se halla inmerso, y de la dirección de su acto (1982, p. 3).

\section{CONCLUSIONES}

Se presentan a continuación los aportes de G. H. Mead - derivados del pragmatismo y de la corriente del interaccionismo simbólico - a los procesos de learning by interacting en los sistemas de innovación, que se hallan en el marco del evolucionismo económico, y a los procesos de creatividad por interacción social.

Los filósofos pragmatistas, y los economistas que estudian los sistemas de innovación, comparten la influencia de las teorías evolucionistas. En particular, las derivadas del pensamiento de Ch. Darwin en el primer caso; y en el segundo, economistas también próximos a las ideas de J. B. Lamarck. El pragmatismo podría brindar sustento epistemológico a las propuestas del evolucionismo económico, como lo ha hecho en el pensamiento del economista Thorstein Veblen (Hodgson, 2007). El método experimental pragmatista, la creatividad derivada de la utilización de la abducción, el principio crítico del falibilismo y la secuencia dinámica de creencias temporales socialmente aceptadas se consideran elementos de interés para aportar a las metodologías de estudio de los
Por otra parte, derivado de las propuestas de Mead sobre el selfy, por ende, del interaccionismo simbólico, surgen dos tipos de aportes para el evolucionismo económico. El self se conforma de un yo y un mí, que representarían la teoría de la acción y la del autocontrol social elaboradas por Mead. En primer lugar, se presentan los aportes que se pueden extraer a partir de dicha teoría.

1) El interaccionismo simbólico podría ser visto solo como una propuesta que promueve la fluida comunicación entre los individuos. De ser esta la conclusión, en el campo de la innovación - como en otros- se debería mantener una continua apertura desde la organización hacia otros agentes del sistema - usuarios, proveedores, etc.-, para recabar sus opiniones. Si bien es innegable la bondad de tal estrategia, esta sería una visión limitada de la obra de Mead. El aporte de su teoría de la acción a los procesos de learning by interacting es además que en toda interrelación cada uno de los participantes es potencialmente creativo.

El yo de cada uno es el componente activo del self que puede resignificar los símbolos antes de emitir el mensaje. Este principio creativo del individuo explica que pueden ser resignificados los símbolos de los objetos en estudio, en los procesos de interrelación entre organizaciones y usuarios, proveedores, empleados, etc. La capacidad creativa se encuentra, en potencia, en cada uno de los individuos, y este elemento debe utilizarse adecuadamente en los procesos innovadores de las organizaciones, aplicables al método de learning by interacting. $\mathrm{Al}$ entrar en interacción con el otro - cliente, proveedor, etc.- se deben crear condiciones para que surjan las ideas preexistentes y las nuevas aproximaciones. En dicha comunicación, el representante de la organización tiene la potencialidad de resignificar el producto y debe desarrollar su propia creatividad. Toda interrelación puede generar aprendizaje, y toda interacción es potencialmente creativa. Dada la acción espontánea del yo, es posible una "creatividad por interacción", de carácter incremental. Frente a la idea de destrucción creativa de Schumpeter (Schumpeter, 1984) relacionada con las innovaciones radicales, los economistas evolucionistas tienden a centrar sus análisis en las innovaciones incrementales, y los aportes teóricos de Mead son un soporte para esta forma de accionar.

2) El segundo componente del self es el mí que representa en el interior del individuo la presencia de la 
conciencia social. Esta presencia interna del otro generalizado lleva a que se proceda reflexivamente antes de iniciar una interacción. De esta forma, en el análisis de Mead, las interacciones sociales resultantes serían de orden autorreguladas, no siendo conflictivas, llevando a descartar la presencia continua de un árbitro externo de carácter hobbesiano (Hobbes, 1999).

Los sistemas de innovación son definidos en la actualidad como espacios de desarrollo de continuos procesos de interacción para generar nuevo aprendizaje. Su actividad central es el aprendizaje, con una comunicación compleja entre las partes interactuantes, en un proceso dialógico de construcción de nuevo conocimiento. Dado que en los procesos de learning by interacting una parte sustancial del conocimiento por intercambiar es de origen tácito, será necesario que los interlocutores tanto en el interior de la organización como con los miembros de otras instituciones del sistema de innovación desarrollen interacciones con un elevado grado de confianza. Compartir e intercambiar conocimientos no codificados requiere que las partes intervinientes asignen al proceso una baja probabilidad de existencia de selección adversa y riesgo moral. En paralelo a las propuestas de Mead, las sociedades cuyos participantes evidencian un comportamiento de autocontrol social elevado demuestran un mayor grado de capital social, de esta forma, entre otros beneficios, se obtiene una mejor relación para desarrollar eficientemente sistemas de innovación (Lundvall, 2005b; Lundvall, Chaminade y Vang, 2009).

\section{REFERENCIAS BIBLIOGRÁFICAS}

1. Arrow, K. J. (1962). The Economic Implications of Learning by Doing. The Review of Economic Studies, 29(3), 155-173.

2. Barrena, S. (2008). Charles S. Peirce:

Razón creativa y educación. Utopia y Praxis Latinoamericana, 13(40). pp. 11-38.

3. Bernstein, R. (1993). El resurgir del pragmatismo. Philosophica Malacitana, 18-19, 11-30.

4. Blumer, H. (1982). El interaccionismo simbólico. Barcelona: Hora Nova.

5. Carabaña,J.y Lamo, E. (1978). La teoría social del interaccionismo simbólico: análisis y valoración crítica. Revista de Investigaciones Sociológicas REIS, 1, 159-203.
6. Cooley, C.H. (1902). Human Nature and the Social Order. Nueva York: Scribner's.

7. Dewey, J. (1903). Democracy in Education. The Elementary School Teacher, 4(4), 193-204.

8. Dewey, J. (1930). What I Believe. Forum, 176-182.

9. Fadiman, C. E. (1990). Living Philosophies. Nueva York: Doubleday.

10. Hobbes, T. (1999/1651). Leviatán. México: Fondo de Cultura Económica.

11. Hodgson, G. M. (2007). Institutions and Individuals: Interaction and Evolution. Organization Studies, 28(1), 95-116.

12. Jacobs, G. (2006). Charles Horton Cooley: Imagining Social Reality. Amherst: University of Massachusetts Press.

13. Joas, H. (1978). G. H. Mead. En D. Kasler (ed.), Klassiker des soziologischen Denkens 2 (pp. 7-39). Múnich: Beck.

14. Joas, H. (1998). El pragmatismo y la teoría de la sociedad. Madrid: Centro de Investigaciones Sociológicas.

15. Johnson, B., Edquist, C. and Lundvall, B-Å. (2003). Economic Development and the National System of Innovation Approach. En First Globelics Conference. Río de Janeiro, Brasil.

16. Lundvall,B.-Å.(1992) National Systems of Innovation. Toward $a$ Ttheory of Innovation and Interactive Learning. Londres. Pinter Publishers.

17. Lundvall,B.-Å.(2005a). National Innovation Systems-Analytical Concept and Development Tool. En DRUID Conference. Copenhagen, Dinamarca.

18. Lundvall,B.-Å.(2005b).Interactive Learning, Social Capital and Economic Performance. En Advancing Knowledge and the Knowledge Economy. Washington D. C.

19. Lundvall,B.-Å.(2007). Innovation Systems: Theory and Policy. En H. Hanusch (ed.), Companion to Neo-Schumpeterian Economics. Londres: Elgar.

20. Lundvall,B.-Å.,Joseph,K.J., Chaminade, C. y Vang, J.(2009). Handbook of Innovation Systems in Developing Countries. Cheltenham: Elgar.

21. Madden, E. y Madden, M. (1952). Chauncey Wright and the Logic of Psychology. Philosophy of Science, 19(4), 325-332.
Sistemas de 
22. Mead, G. H. (1982/1932). Espíritu, persona y sociedad, Barcelona: Paidós Ibérica.

23. Nubiola, J. (2001). La abducción o lógica de la sorpresa. Razón y Palabra, 21. Recuperado de http://www.razonypalabra.org.mx/anteriores/ n21/21_jnubiola.html

Retondaro I

24. Parraviccini, A. (2009). A New Use for an Old Theory. Cognitio-Estudos: Revista Electrónica de Filosofia, 6(2), 110-118.

25. Peirce, C. (1878). How to Make our Ideas Clear. Popular Science Montbly, 12, 286-302.

26. Peirce, C. (1903). Pragmatism as a Principle and Method of Right Thinking: The 1903 Harvard Lectures on Pragmatism. Albany: State University of New York Press.

27. Rescher, N. (2012). Pragmatism: The Restoration of its Scientific Roots. New Jersey: Transaction Publishers.

28. Rizo, M. (2008). Pragmatismo, sociología fenomenológica y comunicología: acción y comunicación en William James y Alfred Schütz. Razón y Palabra, 64, 3. Recuperado de http://www.razonypalabra.org.mx/N/n64/ actual/mrizo.html

29. Rosenberg, N. (1982). Inside the Black Box: Technology and economics. Cambridge: Cambridge University Press.

30. Sánchez de la Yncera, I. (1990). La mirada reflexiva de G. H. Mead sobre la socialidad y la comunicación. Madrid: Centro de Investigaciones Sociológicas.

31. Schumpeter, J. (1984/1942). Capitalismo, socialismo y democracia. Barcelona: Folio.

32. Sini, C. (1999). El pragmatismo. Madrid: Akal.

33. Smith, A. (1997/1759). La teoría de los sentimientos morales. México: Fondo de Cultura Económica.

Panorama I

pp. 62-72।

Volumen 9 |

Número 17।

Julio-diciembre |

2015 I 